\title{
Fatores Ambientais e Genéticos que Influenciam o Desenvolvimento Ponderal até o Desmame de Animais Nelore Criados no Nordeste do Brasil ${ }^{1}$
}

\author{
Stefano Biffani ${ }^{2}$, Raimundo Martins Filho ${ }^{3}$, Andrea Martini ${ }^{4}$, Riccardo Bozzi ${ }^{5}$, Francisco de \\ Assis Melo Lima ${ }^{3}$
}

\begin{abstract}
RESUMO - Dados da Associação Brasileira de Criadores de Zebu (ABCZ), correspondentes a 2977 bezerros da raça Nelore, nascidos no período de 1976 a 1994 e criados em regime de pasto em 11 fazendas dos Estados do Piauí e Ceará, foram analisados para estudar o efeito dos fatores de ambiente e estimar as herdabilidades para a característica peso aos 205 dias de idade (peso a desmama). O modelo matemático incluiu os efeitos fixos de sexo, ano, estação e fazenda de nascimento, a covariável idade da mãe e o efeito aleatório de touro. Os componentes de variância usados para estimar a herdabilidade $\left(\mathrm{h}^{2}\right)$ foram obtidos pelo método da Máxima Verossimilhança Restrita Livre de Derivativa, usando o programa MTDFREML. Os efeitos fixos de sexo, ano e fazenda de nascimento foram significativos e os de estação de nascimento e a idade da mãe ao parto, não-significativos. A média ajustada para o peso à desmama foi de 129,06 $\pm 1,46 \mathrm{~kg}(\mathrm{CV}=17,6 \%)$. Os machos foram, em média, $12 \mathrm{~kg}$ mais pesados que as fêmeas e os animais mais pesados nasceram no ano 1982, com média de peso de 146,54 $\pm 5,22 \mathrm{~kg}$, enquanto os mais leves nasceram no ano 1979, com média de peso de 114,13 $\pm 4,51 \mathrm{~kg}$. As diferenças entre as fazendas foram bastante evidentes, e algumas apresentaram médias muito elevadas em relação à média geral. A herdabilidade para o peso a desmama foi 0,48 $\pm 0,08$.
\end{abstract}

Palavras-chave: bovino Nelore, peso corporal, herdabilidade

\section{Environmental and Genetic Effects Influencing the Ponderal Development up to Weaning Time of Nellore Animals Raised in the Northeastern Brazil}

\begin{abstract}
Growth records from Associação Brasileira de Criadores de Zebu (ABCZ), correspondent to a 2977 Nellore breed calves, born from 1976 to 1994 and raised on pasture in 11 herds from Piauí and Ceará states were analyzed to study the environmental factor effects and to estimate the heritability for the traits weight at 205 days of age (weaning weight). The mathematical model included the fixed effects of sex, year, season and herd of the birth, the covariant age of the dam and the random effect of the sire. The variance components used to estimate the heritability $\left(h^{2}\right)$, were obtained by Derivative Free Restricted Maximum Likelihood, using MTDFREML program. The fixed effects of sex, year and herd of birth were significant, whereas the season of birth and the age of the dam at calving were not significant. Least squares means for the weaning weight was $129.06 \pm 1.46 \mathrm{~kg}(\mathrm{CV}=17.6 \%)$. Males were an average, $12 \mathrm{~kg}$ heavier than females and the heaviest animals were born in the year of 1982, with a mean weight of $146.54 \pm 5.22 \mathrm{~kg}$, while the lightest ones were born in the year of 1979 , with a mean weight of $114.13 \pm 4.51 \mathrm{~kg}$. Differences among herds were evident, and some showed high mean values in relation to the overall mean value. The heritability for weaning weight was $.48 \pm .08$.
\end{abstract}

Key Words: Nellore cattle, corporal weight, heritability

\section{Introdução}

Nos mamíferos, o crescimento pré e pós-natal, principalmente no período até o desmame, é o resultado da ação do seu próprio genótipo e da influência do ambiente materno. Assim, o valor da característica que está sendo observada em um animal representa o valor fenotípico do próprio indivíduo, o qual se compõe de um componente direto (o indivíduo) e de um componente indireto (a mãe)(WILLHAM, 1963; WILLHAM, 1972).

De fato, se por um lado o crescimento de um indivíduo depende do seu próprio genótipo, o qual é constituído por metade dos genes maternos, por outro lado depende da produção de leite e da habilidade materna da mãe, a qual por sua vez depende do seu genótipo e das condições ambientais nas quais os animais estão sendo criados (MEYER, 1992; VAN VLECK et al., 1996). Pode-se, então, afirmar que o peso ao desmame apresenta dupla vantagem, refletindo, por um lado, a habilidade da mãe e, por outro, o princípio da manifestação do mérito próprio do animal para se desenvolver. Além disso, é nessa

\footnotetext{
1 Parte da Dissertação do primeiro autor, apresentada à UFC. Apoio financeiro: CAPES e CNPq.

2 Estudante de Pós-Graduação em Zootecnia da Universidade Federal do Ceará.

3 Professores Adjuntos, Departamento de Zootecnia, CCA/UFC. martins@ufc.br

${ }^{4}$ Professor Titular, Departamento de Ciências Zootécnicas da Universidade de Firenze - Itália.

5 Doutor, Departamento de Ciências Zootécnicas da Universidade de Firenze - Itália. giorgetti@zoot.agr.unifi.it
} 
época que são efetuadas as vendas de bezerros para recria e acabamento; portanto, o ganho econômico depende do peso atingido nessa faixa etária (MINYARD et al., 1965; LÔBO, 1992).

MEYER (1994), para a característica peso ao desmame, relatou que valores entre 0,07 e $0,36 \%$ da variância fenotípica sofrem influência materna, enquanto ROBINSON (1996) encontrou valores entre 0.09 e $0.15 \%$. Isso demonstra que, a essa idade, a influência da mãe é ainda importante, podendo mascarar o efetivo valor genético do indivíduo que está sendo avaliado. ROBINSON e O'ROURKE (1992), trabalhando na Austrália, com três diferentes rebanhos da raça Australian Brahman, na análise preliminar que investigou a influência dos vários efeitos ambientais em diferentes idades, verificaram que entre 60 e $70 \%$ da variação fenotípica foram decorrentes de fatores não-genéticos. No caso do peso ao desmame, $30 \%$ eram atribuídos ao efeito ano de nascimento e $5 \%$, ao sexo.

$\mathrm{O}$ ano de nascimento e, dentro de cada ano, a estação de nascimento são dois efeitos importantes que influenciam o crescimento de um animal, principalmente, se ele e a mãe forem criados em regime de campo, uma vez que os alimentos disponíveis irão depender das condições climáticas vigentes na estação e no ano de nascimento. Na verdade, esses fatores têm, sobre o animal avaliado, efeito indireto durante a primeira fase de vida, que vai do nascer até o desmame, e efeito direto durante a segunda fase. Na primeira fase, é a mãe do bezerro que sofre as influências das condições climáticas e, portanto, indiretamente, também o bezerro é influenciado. Logo após o desmame, o bezerro passa a sofrer maior influência do clima, o que é próprio dos ambientes tropicais, em que são freqüentes ciclos de seca. $\mathrm{O}$ efeito da alimentação está, portanto, intimamente ligado com a estação e o ano de nascimento, sobretudo em um tipo de criação que não prevê nenhuma suplementação alimentar.

CUNDIFF et al. (1966), examinando a importância de diferentes efeitos ambientais sobre o peso ao desmame em animais das raças Hereford e Angus, constataram que os efeitos de sexo, fazenda, raça e idade da mãe ao parto foram significativos, com valor expresso em percentuais da variância fenotípica iguais a 17, $7 \mathrm{e}$ $12 \%$, respectivamente. A idade da mãe foi dividida em 5 classes: até 27 , de 28 até 30, de 31 até 33, de 34 até 39 e de 40 até 45 meses. O peso ao desmame teve crescimento exponencial, aumentando de $22 \mathrm{~kg}$ entre as idades de 27 e 45 meses. Provavelmente esse aumento é resultado das modificações morfo-fisiológicas normais, dos diferentes tecidos e dos vários órgãos, que acontecem durante o crescimento. Isso quer dizer que as novilhas ainda em fase de crescimento produzem bezerros mais leves, devido ao menor desenvolvimento dos órgãos reprodutores, e de menor irrigação do útero, com possível competição pelos nutrientes com o feto. Vacas velhas também produzem bezerros mais leves, porém em decorrência da deficiência de irrigação placentária.

AMARAL et al. (1986) encontraram, em 2670 animais da raça Nelore, peso médio aos 205 dias igual a $166 \pm 0,6 \mathrm{~kg}$. Esse valor representa a média ajustada para os efeitos de sexo, mês e ano de nascimento e idade da vaca ao parto, os quais foram incluídos no modelo estatístico utilizado na análise da variância e que resultaram significativos.

Utilizando os mesmos efeitos ea mesmaraça(Nelore), ELER et al. (1989) relataram que os machos foram, em média, $14 \mathrm{~kg}$ mais pesados que as fêmeas e que os animais mais pesados nasceram também de julho a setembro, com peso médio ajustado de $150 \mathrm{~kg}$.

RIBEIRO et al. (1990), visando determinar a influência dos fatores ambientais sobre os pesos aos 205 dias de 5.390 bezerros Nelore nascidos no Estado da Paraíba, acharam significativos os efeitos de ano e mês de nascimento, sexo do bezerro e idade da mãe ao parto. Os animais que desmamaram com maior peso foram aqueles nascidos em dezembro.

Em 29.032 animais Nelores criados a pasto em 86 fazendas distribuídas, em cinco regiões nos Estados de Minas Gerais, Mato Grosso do Sul e São Paulo, SILVA et al. (1990) verificaram que os machos pesaram, aos 205 dias $8,45 \%$ mais que as fêmeas e a idade de produção máxima das mães foi de 123 meses. MOREIRA et al. (1992), em 549 animais da raça Nelore, criados no Estado do Paraná, calcularam peso médio ajustado pelos efeitos de sexo, ano e dia de nascimento e idade da mãe ao parto, de 158,8 $\pm 0,2 \mathrm{~kg}$. SOUZA e RAMOS (1995), utilizando dados de 2563 animais Nelore, nascidos do período de 1962 a 1983, observaram que os efeitos de touro, vaca, sexo, mês, ano de nascimento foram significativos e a média ajustada de peso ao desmame (210 dias) para esses efeitos foi de $154,40 \pm 23,37 \mathrm{~kg}$. MARTINS FILHO et al. (1997) conduziram estudo sobre os fatores ambientais e genéticos que influem nos pesos e nos ganhos em pesos de bovinos zebú no Estado do Ceará, trabalhando com 8970 registros de animais das raças Nelore, Gir e Guzerá. Os efeitos fixos foram reunidos em grupos contemporâneos, que resultaram altamente 
Rev. bras. zootec.

significativos, tendo sido obtido para a raça Nelore o peso médio ao desmame de $119,77 \pm 21,21 \mathrm{~kg}$.

SOUZA (1997), estudando os pesos ao desmame de 54.248 zebuínos da raça Nelore, distribuídos em oito regiões do centro e norte do Brasil, encontrou valor ajustado igual a $153,33 \pm 0,33 \mathrm{~kg}$, resultando significativos os efeitos de sexo, mês e ano de nascimento, região, fazenda e idade da vaca ao parto.

Os objetivos deste trabalho foram avaliar do desenvolvimento ponderal até o desmame de animais da raça Nelore criados no Estado do Ceará e do Piauí, identificando as fontes de variação não-genética que influem no crescimento, e calcular os componentes de variância por intermédio do método DFREML, para permitir as estimativas dos parâmetros genéticos e ambientais para o peso aos 205 de idade.

\section{Material e Métodos}

Os dados utilizados no presente estudo, obtidos em função do convênio firmado entre a Associação Brasileira de Criadores de Zebú - ABCZ e a Universidade Federal do Ceará - UFC, referem-se a animais da raça Nelore incluídos no sistema de Controle de Desenvolvimento Ponderal (CDP), realizado pela ABCZ. Os dados coletados durante 19 anos (19761994) provêm de 11 fazendas distribuídas nos Estados do Ceará e do Piauí e são o resultado das pesagens efetuadas trimestralmente até os 18 meses de idade, sendo que somente a pesagem ao nascer foi feita pelo criador, enquanto as demais pesagens foram efetuadas por técnicos da $\mathrm{ABCZ}$. $\mathrm{O}$ arquivo geral dos dados continha 5252 observações, relativas a 2598 bezerros e 2654 bezerras, filhos de 167 touros e 2896 vacas, criados em regime de pasto onde receberam suplementação mineral. Os animais tinham acesso livre à pastagem nativa, a qual representava a única forma de alimentação, além da disponibilidade contínua de sal comum e de uma suplementação mineral. Todavia, é importante ressaltar que, em períodos severo de escassez de chuva, os criadores podem suplementar os animais com volumoso de várias origens, sem registrar o fato. Os Estados do Ceará e do Piauí, nos quais estão localizadas as 11 fazendas estudadas, situam-se na subregião semi-árida nordestina, a qual representa aproximadamente $13 \%$ do território brasileiro, com as seguintes características naturais: predominância de solos rasos, sobretudo na porção norte; predominância de relevo ondulado; vegetação de caatinga; irregularidade pluviométrica; e ocorrência de secas periódicas. Para efeito da análise dos dados, os meses do ano foram agrupados em: janeiro, fevereiro - junho (estação chuvosa = 1) e julho - dezembro (estação seca $=2$ ), de acordo com a distribuição das chuvas durante o ano na região Nordeste do Brasil.

Do arquivo geral foram utilizadas somente as informações relativas aos pesos ao desmame, eliminando-se também os reprodutores que tinham menos de seis filhos. A partir dessas restrições, foi obtido um arquivo com 2977 observações, relativas a 1433 machos e 1544 fêmeas filhos de 106 touros. Todos os pesos foram ajustados às idades-padrão de 205 dias, segundo as fórmulas descritas por LÔBO (1992).

O modelo estatístico geral utilizado no procedimento GLM do programa SAS (SAS, 1996) foi:

$$
\begin{aligned}
& \quad Y_{j i l k m n}=\mu+F_{i}+R_{i j}+A_{k}+E_{l}+S_{m}+b_{1}\left(I_{i j k l m n}-\overline{\mathrm{I}}\right) \\
& +b_{2}\left(I_{i j k l m n}-\mathrm{I}\right)^{2}+e_{i j k l m n} \\
& \quad \text { em que }
\end{aligned}
$$

$Y_{j i l k m n}=$ peso aos 205 dias de idade do n-esimo filho do reprodutor $j$ dentro da fazenda $i$, nascido na estação $l$ do ano $k$, do sexo $m$;

$\mu=$ média geral para as características estudadas;

$F_{i}=$ efeito fixo da fazenda;

$R_{i j}=$ efeito aleatório do reprodutor $j$ dentro da fazenda $i$

$A_{k}=$ efeito do ano do nascimento $k$;

$E_{l}=$ efeito da estação $l$;

$S_{m}=$ efeito do sexo da cria $m$;

$I_{i j k l m n}=$ idade da vaca ao parto;

$b_{1}=$ coeficiente de regressão para idade linear;

$b_{2}=$ coeficiente de regressão para Idade quadrática; e

$e_{j i l k m n}=$ erro aleatório, normal, independentemente distribuído com média zero e variância $\sigma^{2}$.

As estimativas dos componentes de variância foram obtidas pelo método da Máxima Verossimilhança Restrita não Derivativa - DFREML, utilizando-se o programa MTDFREML, (BOLDMAN et al., 1993). Visto que não houve informações do parentesco, foi utilizado um modelo de touro (Sire Model), assumindo que não havia reprodutores parentes.

Usando uma notação matricial, o modelo de touro resulta ser:

$$
Y=X \beta+Z u+e
$$

em que

$Y=$ vetor dos pesos ou ganhos em pesos;

$\beta=$ vetor dos efeitos fixos;

$u=$ vetor dos efeitos aleatórios;

$e=$ vetor dos efeitos residuais aleatórios;

$X=$ uma matriz de incidência da ordem $n x p$, que 
associa cada observação a cada efeito fixo;

$Z=$ matriz da ordem $n x t$ que associa cada touro as observações de sua progênie;

os valores esperados $E$ são:

$$
E\left[\begin{array}{l}
y \\
s \\
e
\end{array}\right]=\left[\begin{array}{l}
X b \\
0 \\
0
\end{array}\right]
$$

com matriz de variância e covariância

$$
\operatorname{Var}\left[\begin{array}{l}
y \\
u \\
e
\end{array}\right]=\left[\begin{array}{lll}
\mathrm{ZZ} \sigma_{\mathrm{s}}^{2}+\mathrm{I} \sigma_{e}^{2} & \mathrm{Z} \sigma_{\mathrm{s}}^{2} & 0 \\
& \mathrm{I} \sigma_{\mathrm{s}}^{2} & 0 \\
\text { Simétrica } & & 0
\end{array}\right]
$$

em que as I são as matrizes de identidade.

Os componentes de variância obtidos foram utilizados para estimar a herdabilidade $\left(\mathrm{h}^{2}\right)$, calculada pela correlação intra classe de meio-irmãos paternos. Os erros-padrão das estimativas foram calculados de acordo com a fórmula proposta por Swiger (1964).

\section{Resultados e Discussão}

A média ajustada para o peso ao desmame (P205) foi de $129,06 \pm 1,46 \mathrm{~kg}$ com coeficiente de variação igual a $17,6 \%$. O valor encontra-se abaixo dos resultados obtidos por todos os autores revisados, à exceção do citado por MARTINS FILHO et al. (1997), que, no Estado do Ceará, obtiveram valor do peso ao desmame de $119,77 \pm 21,21 \mathrm{~kg}$.

Os resultados resumidos da análise da variância são apresentados na Tabela 1 .

Das fontes de variações analisadas, foram significativos ( $\mathrm{P}<0,001)$ o efeito do touro (aleatório) e os efeitos de fazenda, ano de nascimento e sexo (fixos). Não houve efeito significativo da estação de nascimento e da idade da mãe ao parto, utilizada como covariável. O efeito de touro foi significativo, como era esperado, e confirmado em todos os trabalhos revisados, já que $50 \%$ dos genes que um indivíduo possui, provêm do reprodutor, sendo, portanto, razoável admitir que filhos de animais geneticamente diferentes apresentem desempenhos diferentes. Esse efeito entrou no modelo como aleatório e hierarquizado, uma vez que a estrutura dos dados disponíveis provocou confudimento com o efeito de fazenda. As médias ajustadas para o sexo (Tabela 2) foram iguais a $133,18 \pm 1,47 \mathrm{~kg}$ para os machos e $126,01 \pm 1,43 \mathrm{~kg}$ para as fêmeas, o que está de acordo com os resultados encontrados por ROBINSON e O'ROURKE (1992) e PLASSE et al. (1995), na raça Brahman, e por OLIVEIRA-FILHO et al. (1980), ELER et al. (1989), RIBEIRO et al. (1990), SILVA et al. (1990), MOREIRA et al. (1992), SOUZA e RAMOS (1995) e MARTINS FILHO et al. (1997), na raça Nelore, confirmando a superioridade dos machos sobre as fêmeas, provavelmente, devido à atividade fisiológica e hormonal maior nos machos, o que levaria à capacidade maior de amamentação e, então, ao crescimento mais rápido.

$\mathrm{O}$ ano de nascimento do bezerro foi outro efeito ambiental que influenciou significativamente o peso aos 205 dias dos animais $(\mathrm{P}>0,001)$. Este resultado está de acordo com os encontrados por OLIVEIRAFILHO et al. (1980), AMARAL et al. (1980), ELER et al. (1989), RIBEIRO et al. (1990), SILVA et al. (1990), MOREIRA et al. (1992), SOUZA e RAMOS (1995), em raças zebuínas.

As médias ajustadas obtidas e o número de observações disponíveis por ano de nascimento são apresentados na Tabela 2.

Os animais mais pesados nasceram no ano 1982, com média de $146,54 \pm 5,22 \mathrm{~kg}$, e os mais leves, no ano 1979 , com média igual a $114,13, \pm 4,51$. As diferenças de peso verificadas entre os vários anos são explicadas pelas diferentes condições climáticas próprias de cada ano, as quais se refletem em diferentes disponibilidade de alimento. Na verdade, nessa fase de crescimento, em que o bezerro se encontra ainda mamando, a menor ou maior disponibilidade de alimento influencia diretamente a vaca e só indiretamente ao bezerro, já que o seu alimento principal é o leite. Essa situação é própria de ambientes como o do Nordeste do Brasil, onde há ocorrência de ciclos de seca e, portanto, a produção de alimento não é constante, seja no que se refere à quantidade ou à qualidade.

O comportamento sinusóidal do peso médio por ano ao redor da média geral é conseqüência direta da variabilidade, devido às condições climáticas.

O efeito fixo de fazenda, conforme a análise da variância (Tabela 1), exerceu efeito significativo $(\mathrm{P}<0,001)$, confirmando resultados já encontrados por ELER et al. (1989) e SOUZA (1997), os quais haviam utilizado esse efeito no modelo da análise.

No presente estudo foram utilizadas 11 fazendas e as médias ajustadas dos pesos e o número de observações são apresentadas na Tabela 2, sendo as fazendas identificadas pelo número de registro na $\mathrm{ABCZ}$. As diferenças foram bastante evidentes, com 
Rev. bras. zootec.

Tabela 1 - Resumo da análise de variância para o peso ao desmame

Table 1 - Summary of the analysis of variance for weaning weight Fonte de variação gl Quadrado médio

Source of variation $\quad d f \quad$ Mean square

Touro dentro de fazenda $\quad 117 \quad 2009,57^{* *}$

Sire within farm

Sexo $135369,80 * *$

Sex

Ano de nascimento $18 \quad 6685,05^{* *}$

Year of birth

Fazenda $\quad 10 \quad 24087,23 * *$

Herd

Estação de nascimento

Season of birth

Idade da vaca ao parto, linear

Cow age at calving, linear

Idade da vaca ao parto, quadrático

Cow age at calving, quadratic

Erro

Error

2827

$\mathrm{R}^{2}$

$* * \mathrm{P}<0,001$.

algumas fazendas apresentando médias muito elevadas em relação à média geral, como as fazendas 39 , 3127 e 3438 com médias iguais a 153,$17 ; 157,52$; e $151,42 \mathrm{~kg}$, respectivamente. Estas diferenças podem ser atribuídas, principalmente, às diferenças de manejo, condições climáticas e solos das propriedades, mas podem ter também componente genético, que, além das diferenças normais na composição genética existentes entre rebanhos diferentes, poderia ser o resultado de um processo seletivo exercido pelos criadores. Deve-se ressaltar, também, que o número de observações disponíveis pode ter influenciado os resultados, já que os pesos menores foram observados nas fazendas com o menor número de dados (fazenda 3401 e 6037). Quando se excluiu do conjunto os dados referentes as médias dessas fazendas, a média entre as fazendas foi aumentada em 4,3\%.

Contrariamente à maioria dos trabalhos revisados, como os de ELER et al. (1989) e SILVA et al. (1990), neste estudo não foi encontrado efeito significativo da estação de nascimento sobre o peso aos 205. Este resultado deve ser analisado com atenção, pois poderia ser conseqüência de dois aspectos diferentes. O peso aos 205 dias foi escolhido como idade padrão para delimitar as diferentes fases do crescimento, isto é, a primeira fase que começa ao nascer e vai até o desmame e a segunda que vai do desmame até a maturidade. Isso significa que não há segurança que aos 205 dias de idade o bezerro tenha sido realmente desmamado, principalmente se os animais
Tabela 2 - Médias ajustadas e número de observações de acordo com o sexo, o ano e a fazenda de nascimento, para o peso aos 205 dias de idade

Table 2 - Least square means for calf weaning weight according to sex, year and herd of birth

\begin{tabular}{|c|c|c|}
\hline $\begin{array}{l}\text { Efeito } \\
\text { Effect }\end{array}$ & $\begin{array}{c}\mathrm{N}^{\mathrm{O}} \text { das observação } \\
\text { N. of observation }\end{array}$ & $\begin{array}{c}\text { Média } \pm \text { e.p. }(\mathrm{kg}) \\
\text { Mean } \pm \text { s.d. }\end{array}$ \\
\hline \multicolumn{3}{|l|}{ Sexo } \\
\hline \multicolumn{3}{|l|}{ Sex } \\
\hline $\begin{array}{l}\text { Macho } \\
\text { Male }\end{array}$ & 1433 & $133,18 \pm 1,47$ \\
\hline $\begin{array}{l}\text { Fêmea } \\
\text { Female }\end{array}$ & 1544 & $126,01 \pm 1,43$ \\
\hline \multicolumn{3}{|l|}{ Ano } \\
\hline \multicolumn{3}{|l|}{ Year } \\
\hline 1976 & 94 & $129,18 \pm 5,47$ \\
\hline 1977 & 136 & $129,17 \pm 4,92$ \\
\hline 1978 & 184 & $117,74 \pm 4,55$ \\
\hline 1979 & 186 & $114,13 \pm 4,51$ \\
\hline 1980 & 119 & $117,13 \pm 4,96$ \\
\hline 1981 & 94 & $115,13 \pm 4,70$ \\
\hline 1982 & 51 & $146,54 \pm 5,22$ \\
\hline 1983 & 39 & $146,17 \pm 6,65$ \\
\hline 1984 & 68 & $127,69 \pm 4,09$ \\
\hline 1985 & 143 & $118,52 \pm 2,74$ \\
\hline 1986 & 258 & $138,76 \pm 2,57$ \\
\hline 1987 & 237 & $134,97 \pm 2,37$ \\
\hline 1988 & 252 & $132,90 \pm 2,29$ \\
\hline 1989 & 181 & $122,18 \pm 2,49$ \\
\hline 1990 & 266 & $126,32 \pm 2,49$ \\
\hline 1991 & 186 & $136,46 \pm 2,74$ \\
\hline 1992 & 144 & $145,44 \pm 3,40$ \\
\hline 1993 & 266 & $133,43 \pm 3,24$ \\
\hline 1994 & 73 & $130,48 \pm 4,69$ \\
\hline
\end{tabular}

Fazenda

Herd

\begin{tabular}{lrr}
39 & 158 & $153,17 \pm 3,48$ \\
235 & 170 & $135,91 \pm 3,52$ \\
265 & 214 & $130,20 \pm 3,72$ \\
273 & 558 & $124,20 \pm 3,42$ \\
1670 & 369 & $125,14 \pm 2,76$ \\
2988 & 93 & $116,79 \pm 3,53$ \\
2989 & 189 & $122,38 \pm 3,96$ \\
3127 & 791 & $157,52 \pm 2,50$ \\
3401 & 17 & $100,59 \pm 6,54$ \\
3438 & 399 & $151,42 \pm 2,94$ \\
6037 & 19 & $108,24 \pm 6,37$ \\
\hline
\end{tabular}

foram criados a campo, sem controle direto. Portanto, nessas condições, é muito elevada a possibilidade de se encontrarem bezerros com idade superior aos 205 idades e mamando. Na maioria das fazendas estudadas, os animais vivem em regime de pasto durante o ano todo, e só em poucas existe controle mais acurado e constante da época do desmame. Outro aspecto que deve ser considerado é que a utilização de somente duas estações, chuvosa e seca, não consegue detectar as prováveis diferenças existentes ao longo do ano, ainda mais quando se sabe que as 
condições climáticas das regiões do nordeste do Brasil estão sujeitas à variabilidade anual, com estação chuvosa não bem definida. A utilização das duas estações não garante que essa variabilidade seja detectada e, talvez, seja mais indicada a utilização dos trimestres de nascimento ou até do mês de nascimento.

Idade da mãe - efeito utilizado como covariável não resultou significativo neste estudo, testando os efeitos linear e quadrático. Neste caso, o resultado obtido discorda da maioria dos trabalhos revisados, os quais relatam a existência de uma relação, geralmente de tipo quadrático, entre a idade da mãe e o peso ao desmame, sendo que as novilhas e as vacas mais velhas desmamam bezerros mais leves. A influência da idade da mãe decorre da habilidade materna e, principalmente, da sua capacidade produtiva de leite. Pelos resultados obtidos, parece não ter ocorrido diferença entre as vacas mais novas e as mais velhas ou que, por alguma razão, essa diferença resultou mascarada. Uma explicação para esse comportamento é que as condições climáticas e as formas de manejo e criação poderiam ter diminuído a potencialidade produtivas das vacas, equilibrando todas as matrizes e mascarando a verdadeira habilidade materna delas. A idade média ao parto das vacas foi de $2984 \pm 1347$ dias, o que resulta em idade superior aos oito anos, e apresentou coeficiente de variação de $45 \%$, valor mínimo de 807 dias ( 2 anos e 3 meses) e valor máximo de 6800 dias (18 anos e 6 meses). O valor médio é bastante elevado, mas a visualização gráfica da distribuição das diferentes classes de idade pode ajudar a entender melhor o comportamento da variável (Figura 1).

Como pode ser observado, as classes de idade

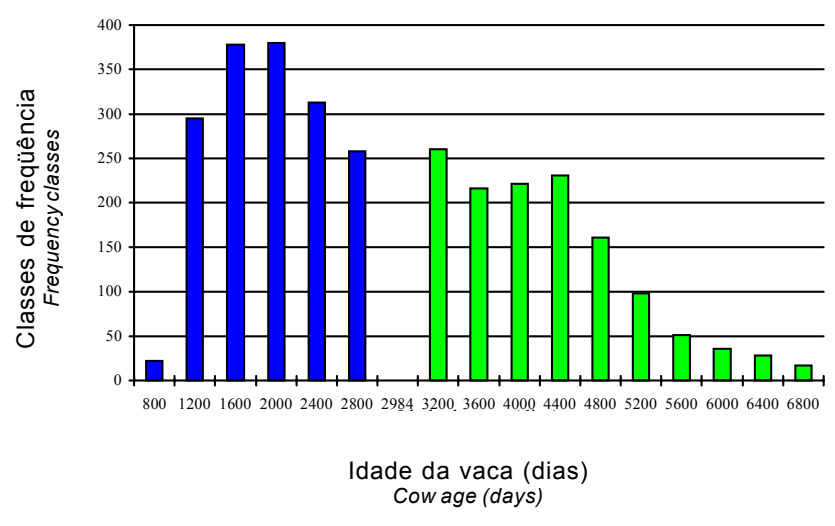

Figura 1 - Distribuição das classes de idade das vacas Figure 1 - Distribution of the age classes of cows. não têm distribuição normal bem definida, mas podem ser identificadas duas concentrações de valores: à esquerda do valor médio (2984 dias), as vacas com idade menor que oito anos, e à direita, as vacas mais velhas (idade $\cong 10$ anos). Segundo MARIANTE et al. (1978) e CUNDIFF et al. (1966), os bezerros que desmamam mais pesados são aqueles filhos das vacas com idade entre 5 e 8 anos. No caso do presente estudo, a idade média muito elevada e a distribuição das classes de freqüência poderiam ter mascarado a maior potencialidade produtiva das vacas na faixa etária de 5 a 8 anos.

A herdabilidade para o peso aos 205 dias de idade foi $0,48 \pm 0,08$. Este resultado se encontra dentro do intervalo de valores obtidos por MOHIUDDIN (1993) e MERCADANTE et al. (1995), os quais foram 0,06-0,63 e 0,05-0,58, respectivamente. Estes valores, comparados aos encontrados por outros autores na mesma raça, se situam em posição intermediária, sendo que ROSA et al. (1986) acharam valores pouco maiores (0,58), enquanto RIBEIRO et al. (1990), LÔBO et al. (1993), ELER et al. (1989) e MARTINS FILHO et (1997), valores menores, 0,16; 0,17; 0,12; 0,24 ; e 0,17 , respectivamente. No caso desses últimos autores, as estimativas, à exceção dos valores obtidos por MARTINS FILHO et al. (1997), são oriundas de rebanhos do sul e sudeste do Brasil, que, há vários anos, estão sujeitos a processo de seleção, o qual tem como conseqüência a diminuição da variabilidade genética entre os indivíduos pertencentes à mesma população (FALCONER, 1989).

Nas condições de criações do Nordeste brasileiro, a pressão de seleção não tem a mesma magnitude que a do sul do país, o que pode resultar em maior variabilidade do componente genético aditivo. CUNHA (1997), avaliando 769 bezerros Nelores criados no Estado do Ceará, encontrou valor de 0,30 \pm 0,10. MARTINS et al. (1996), utilizando 375 informações de bezerros nascidos no Estado do Maranhão, calcularam valor de $0,29 \pm 0,18$. O número não muito elevado das informações disponíveis, se comparado ao do presente estudo (2977), pode explicar as diferenças para menos nos valores encontrados por esses autores.

De forma geral, os valores de herdabilidade obtidos para o peso ao desmame são de magnitude média, mas deve ser levado em consideração que esses valores contêm o efeito materno. Portanto, a utilização destes em um programa de seleção só é indicada se pelo menos estimativa for, como no presente estudo, resultado de um modelo de touro. 


\section{Conclusões}

As variações anuais dos pesos poderiam ser diminuídas pela melhoria da qualidade e quantidade do alimento fornecido aos animais, além dos outros aspectos do manejo em geral, o que proporcionaria aumento de peso e resultaria em maior ganho econômico para o criador.

As diferenças decorrentes do sexo, com a comprovada superioridade em peso dos machos, sugerem que possa ser utilizada alimentação diferenciada entre machos e fêmeas, visando diminuir o tempo necessário para alcançar o peso adequado, para o abate, no caso dos machos, e para o acasalamento, no caso das fêmeas.

A herdabilidade estimada para a característica foi de magnitude média a alta, porém, no caso do peso aos 205 dias de idade, sendo provável a ocorrência da elevada influência materna, não seria indicada a sua utilização como critério único de seleção.

\section{Referências Bibliográficas}

AMARAL, C.O. LÔBO, R.B., DUARTE. F.A.M. et al. Coeficientes de herdabilidade e correlação genética entre características de crescimento em bovinos da raça Nelore. In: REUNIÃO ANUAL DA SOCIEDADE BRASILEIRA DE ZOOTECNIA, 23, 1986, Campo Grande-MS. Anais... Campo Grande: SBZ, 1986. p 309.

BOLDMAN, K.G., KRIESE, L.A., VAN VLECK, D.L. et al. 1993. A manual for use of MTDFREML- a set of programs to obtain estimates of variances and covariances. ARS, USDA.

CUNDIFF, L.V., WILLHAM, R.L., PRATT, C.A. 1966. Effects of certains factors and their two-way interactions on weaning weigth in Beef cattle. J. Anim. Sci., 25:972-982.

CUNHA, R.M. Estudo genético-quantitativo de pesos e ganhos em pesos em bovinos da raça Nelore na fase de aleitamento no Estado do Ceará. Fortaleza, CE. Fortaleza: 1997. 80p. Dissertação (Mestrado em Zootecnia) - Universidade Federal do Ceará, 1997.

ELER, J.P., LÔBO, R.B., ROSA, A.N. 1989. Influência de fatores genéticos e de meio em pesos de bovinos da raça Nelore criados no Estado de São Paulo. R. Soc. Bras. Zootec., 18(2):103-111.

FALCONER, D.S. 1989. Introduction to Quantitative Genetics. 3. ed. Longman Scientific and Technical.

LÔBO, R. B. 1992. Programa de melhoramento genético da raça Nelore, 2. ed., Ribeirão Preto - SP: Universidade de São Paulo.

MARIANTE, A., HARGROVE, D. D., KOGER, M. et al. 1978. Factors Affecting Growth of Nelore Cattle in Central Brazil. J. Anim. Sci., 46:6 (suppl.).

MARTINS FILHO, R., LOBO, R.N.B., LIMA, F.A.M. et al. Parâmetros genéticos e fenotípicos de pesos e ganhos em pesos de bovinos zebus no estado do Ceará. In: REUNIÃO ANUAL DA SOCIEDADE BRASILEIRA DE ZOOTECNIA, 34,1997, Juiz de Fora, MG. Anais... Juiz de Fora: SBZ, 1997. p248-250.
MARTINS, G.A., MARTINS-FILHO, R., LOBO, R.N.B. Fatores genéticos e de ambiente que influenciam o peso à desmama em bovinos da raça Nelore. In: REUNIÃO ANUAL DA SOCIEDADE BRASILEIRA DE ZOOTECNIA, 33, 1996, Fortaleza-CE. Anais... Fortaleza: SBZ, 1996, p181-183.

MERCADANTE, M.E.Z., LÔBO, R.B., BORJAS, A. et al. 1995. Parámetros genéticos para características de crecimento en zebuínos de carne. Archivos Latinoamericanos de Prod. Anim., 3(1):45-89.

MEYER, K. 1994. Estimates of direct and maternal correlations among growth traits in Australian beef cattle. Lvst. Prod. Sci., 38:91-105.

MEYER, K. 1992. Variance components due to direct and maternal effects for growth traits of Australian beef cattle. Lvst. Prod. Sci., 31:179-204.

MINYARD, J.A., DINKEL, C.A. 1965. Heritability and repeatabilitynof weaning weigth in beef cattle. J. Anim. Sci., 24:1072-1074.

MOHIUDDIN, G. 1993. Estimates of genetic and phenotypic parameters of some performance traits in beef cattle. Anim. Breeding Abst., 61(8):495-522.

MOREIRA, H.L.M., RIBEIRO, R.P., RORATO, P.R. et al. Fatores genéticos e de ambiente que afetam os pesos ao nascer e a desmama de terneiros Nelore. In: REUNIÃO ANUAL DA SOCIEDADE BRASILEIRA DE ZOOTECNIA, 29,1992, Lavras, MG. Anais... Lavras: SBZ, 1992. p457.

OLIVEIRA-FILHO, E.B., DUARTE, F.A.M. Efeitos genéticos e não genéticos sobre o ganho de peso de bovinos Nelore do nascimento aos 550 dias de idade. In: REUNIÃO ANUAL DA SOCIEDADE BRASILEIRA DE ZOOTECNIA, 17, 1980, Fortaleza-CE. Anais... Fortaleza: SBZ, 1980, p.176.

PLASSE, D., FOSSI, H., HOOGESTEIJN, R. et al. 1995. Growth of $\mathrm{F}_{1}$ Bos Taurus x Bos Indicus beef cattle in Venezuela. I. Weights at birth, weaning and 18 months. J. Anim. Breeding and Genetics, 112:117-132.

RIBEIRO, M.N., PIMENTA-FILHO, E.C. Estimativas de parâmetros genéticos dos pesos ao nascer e ao desmame de bezerros Nelore. In: REUNIÃO ANUAL DA SOCIEDADE BRASILEIRA DE ZOOTECNIA, 27, 1990, Campinas, SP. Anais... Campinas: SBZ. 1990. p 463.

RIBEIRO, M.N., PIMENTA-FILHO, E.C. Estimativas de parâmetros genéticos dos pesos ao nascer e ao desmame de bezerros Nelore. In: REUNIÃO ANUAL DA SOCIEDADE BRASILEIRA DE ZOOTECNIA, 27, 1990, Campinas, SP. Anais... Campinas: SBZ. 1990. p463.

ROBINSON, D.L. 1996. Estimation and interpretation of direct and maternal genetic parameters for weights of Australian Angus cattle. Livs. Prod. Sci., 45:1-11.

ROBINSON, D.L., O'ROURKE, P.K. 1992. Genetic parameters for liveweights of beef cattle in the tropics. Austr. J. Agric. Res., v. 43, p 1297-1305.

ROSA, A.N., SILVA, L.O.C., NOBRE, P.R.C. 1986. Avaliação do Desempenho de Animais Nelore em Controle de Desenvolvimento Ponderal No Estado do Mato Grosso do Sul, Brasil. R. Soc. Bras. Zootec., 15(6):515-532.

SILVA, L.O.C., MILAGRES, J.C., REGAZZI, A.J. et al. Efeitos genéticos e de ambiente sobre os pesos aos 205 (P205) e 365 (P365) dias de idade, em animais Nelore. In: REUNIÃO ANUAL DA SOCIEDADE BRASILEIRA DE ZOOTECNIA, 27, 1990, Campinas, SP. Anais... Campinas: SBZ. 1990.p 476.

SOUZA, J.C. Interação genótipo $\mathrm{x}$ ambiente sobre o peso ao desmame de zebuínos da raça Nelore no Brasil. Botucatu, SP: 
1997. 70p. Tese (Doutorado em Melhoramento Genético) Universidade Estadual Paulista, 1997.

SOUZA, J.C., RAMOS, A.A. 1995. Efeitos de fatores genéticos e do meio sobre os pesos de bovinos da raça Nelore. $R$. Soc. Bras. Zootec., 24(1):165-170.

SAS STATISTICAL ANALYSIS SYSTEM. 1996. User's guide: Statistics. Version 6.11, NC; SAS Institute.

SWIGER, L.A., HARVEY, W.R., EVERSON, D.O. et al. 1964. The variance of intraclass correlation involving groups with one observation. Biometrics, 20:818-826.

VAN VLECK, L.D., GREGORY, K.E., BENNETT, G.L. 1996. Direct and maternal genetic covariances by age of dam for weaning weight. J. Anim. Sci.., 74:1801-1805.
WILLHAM, R.L. 1963. The covariance between relatives for characters composed of components contributed by related individuals. Biometrics, 19:18-27.

WILLHAM, R.L. 1972. The role of maternal effects in animal breeding: III. Biometrical aspects of maternal effects in animals. J. Anim. Sci.., 35:1288-1293.

Recebido em: 29/09/98

Aceito em: 08/01/99 